\title{
Comprehensive Evaluation of General Education in University Based on Grey Relational Analysis Model
}

\author{
Wang Jiayang ${ }^{1, ~ *, ~ S h a o g u i ~} \mathrm{Wu}^{2}$, Wei Liu ${ }^{1}$ \\ ${ }^{1}$ College of Resources and Environment, Chengdu University of Information Technology, Chengdu, China \\ ${ }^{2}$ College of Chemistry and Materials Science, Sichuan Normal University, Chengdu, China
}

Email address:

goodwji foxmail.com (Wang Jiayang), wsgchem $a$ foxmail.com (Shaogui Wu), 4340641@qq.com (Wei Liu)

${ }^{*}$ Corresponding author

\section{To cite this article:}

Wang Jiayang, Shaogui Wu, Wei Liu. Comprehensive Evaluation of General Education in University Based on Grey Relational Analysis Model. Teacher Education and Curriculum Studies. Vol. 5, No. 1, 2020, pp. 1-6. doi: 10.11648/j.tecs.20200501.11

Received: December 11, 2019; Accepted: December 30, 2019; Published: February 11, 2020

\begin{abstract}
A preliminary evaluation system of general education teaching quality in universities was established based on Bloom's objective classification method from four aspects: teaching objectives, teaching content, implementation process and assessment methods, in view of the impersonality of the evaluation and assessment of teaching quality, the difficulty of evaluation for the supervisory department in teaching, and the difficulty of the refinement and quantification of teaching achievements. A grey relational analysis method was used to establish the evaluation criteria, and then the evaluation model of teachers' general education quality was established. The index system and evaluation model were applied to evaluate many young teachers' education quality in a university. The evaluation results show that: the evaluation system can be used to evaluate the teaching ability of general courses of young teachers, rank them comprehensively, and find out the aspects to be improved. According to the teaching evaluation results, teachers can analyze their ranking among peers, and find out their own advantages and disadvantages. It has theoretical and practical significances for improving the teaching quality of general courses in universities.
\end{abstract}

Keywords: Teaching Evaluation, General Education Curriculum, Comprehensive Evaluation, Grey Relational Analysis

\section{Introduction}

General education aims to make the educated become a "complete" person with subjective and comprehensive basic concepts through the cultivation of basic knowledge, civic awareness, sound personality and some non-professional practical abilities [1-3]. The purpose is to form a comprehensive knowledge, bridging professional education and non-professional education, so that the educated can get free and comprehensive development in knowledge, personality, emotion, reason and other aspects. Since the 1990s, general education has been paid more and more attention in colleges and universities [4-5]. As a complementary and integrated form of education with professional education, general education in China has been developed in recent years. A new round of scientific and technological revolution and industrial revolution have become a trend. The concept of professional counterpart has been gradually weakened, and the general ability and comprehensive ability have attracted much attention [6].

However, the general education in China's colleges and universities has not been carried out for a long time, and the related research on the evaluation of general education is still relatively rare. The work on teaching evaluation has just begun, and most universities still cannot distinguish it from traditional courses [7-10]. Therefore, in view of these problems that the evaluation and assessment of teachers' teaching quality in colleges and universities are not objective, it is difficult for the supervision department to evaluate the teaching quality, and it is difficult to refine and quantify the teaching results of teachers [11-15]. The Bloom Target Classification method [16-17] is used to establish a set of evaluation system for the teaching quality of general education courses in colleges and universities from four aspects of teaching objectives, teaching contents, implementation process and assessment method.

An effective evaluation method is an important guarantee 
for improving the evaluation level. Grey correlation analysis provides a quantitative measure of a system's developmental change situation, which is very suitable for dynamic process analysis. A measure of the magnitude of the association between two systems, which varies over time or from different objects, is called correlation degree. The grey system theory puts forward the concept of gray correlation analysis for each subsystem, and intends to seek the numerical relationship between subsystems (or factors) in the system through certain methods. In the process of system development, if the trends of the two factors are consistent, that is, the value of synchronous change degree is high, which means that the two factors are highly correlated; otherwise, the value is low. Therefore, the grey correlation analysis (GCA) method is based on the degree of similarity or dissimilarity between the development trends of factors, that is, the "grey correlation degree" as a method to measure the degree of association between factors [18].

With the development of grey system theory, gray correlation analysis has been widely used in environmental quality analysis, ecological evaluation, management research and other fields [18-21]. By establishing a grey relational model, the gray relation can be quantified, sequenced and manifested, which can provide an important technical analysis method for the modeling of complex systems [21]. The grey relational analysis method can calculate the degree of association between the index value to be evaluated and different standards, and compare the geometric relationship of the statistical sequence to measure the degree of relationship between multiple factors of the system.

In this work, the GCA method was employed to evaluate the teaching quality of university general education courses. The evaluation criteria were setup by using the grey relation analysis method, and then the evaluation model of teachers' general teaching quality was also established. We applied the index system and evaluation model to analyze many young teachers in a university, realizing the quantitative evaluation of general education teaching quality, deepening the research of general education quality evaluation, and improving the teaching quality of general courses, which is favorable for the improvement of the quality of general education.

\section{Comprehensive Evaluation Model Based on GRA}

The basic idea of GRA is to judge correlation (tightness, relevance) by calculating the gray correlation degree. $0<r\left(x_{0}\right.$, $\left.x_{\mathrm{i}}\right) \leq 1$. The closer its value is to 1 , the more relevant the two factors are. The calculation steps of gray correlation are as follows:

(1) The initial values of each sequence are,

$$
\left.X_{i}^{\prime}=X_{i} / x_{i}(1)=x_{i}^{\prime}(1), x_{i}^{\prime}(2), \ldots x_{i}^{\prime}(n)\right) i=0,1,2, \ldots, m
$$

The values of difference sequence are given by,

$$
\Delta_{i}(k)=\left|x_{0}^{\prime}(k)-x_{i}^{\prime}(k)\right|
$$

Where, $i=1,2, \ldots m ; k=1,2, \ldots, n ; ; x_{0}^{\prime}(k)$ is a reference sequence and $x_{i}^{\prime}(k)$ is the other sequence.

(2) Maximum and minimum differences between two levels are given by,

$$
\begin{gathered}
\Delta_{M}=\max _{i} \max _{k} \Delta_{i}(k) \\
\Delta_{m}=\min _{i} \min _{k} \Delta_{i}(k)
\end{gathered}
$$

(3) Correlation coefficient:

$$
r\left(x_{0}^{\prime}(k), x_{i}^{\prime}(k)\right)=\frac{\Delta_{m}+\xi \Delta_{M}}{\Delta_{i(k)}+\xi \Delta_{M}}
$$

Where, $\xi \in(0,1) ; i=1,2, \ldots m ; k=1,2, \ldots, n$.

(4) Gray correlation degree is calculated by,

$$
r\left(X_{0}, X_{i}\right)=\frac{1}{n} \sum_{k=1}^{n} r_{0 i}\left(x_{0}^{\prime}(k), x_{i}^{\prime}(k)\right)
$$

The larger the correlation degree is, the more consistent the change trend of the reference sequence $x_{0}^{\prime}(k)$ and other sequence $x_{i}^{\prime}(k)$ is. In short, a larger correlation degree denotes a better evaluation object.

\section{Teaching Quality Evaluation Index System of General Courses}

The evaluation of teaching quality is a systematic project, and the factors affecting teaching quality involve many aspects. Based on the analysis of the characteristics of general education courses and combined with the actual situation of teaching evaluation in China, we established an evaluation index system of teaching quality of general education courses in universities adopted an improved Benjamin Bloom's goal classification theory, including four aspects: teaching goal, teaching content, implementation process and evaluation method, as shown in Table 1.

\subsection{Evaluation of Teaching Objectives of General Courses}

Due to the particularity of general education courses, which belong to six fields: basic skills, literature and art, philosophy and Social Sciences, history and culture, natural sciences, and moral ethics and value orientation, it is particularly important to consider the composition and proportion of each subject. Meanwhile, the general education courses need to reflect the spirit of humanity and science and its expression, and it is also an important measurement index when examining the curriculum design objectives. Therefore, we constructed the evaluation system of general course teaching goal using an improved theory of Bloom's goal taxonomy. According to Bloom, it is very important to determine a goal, and then we must choose the "content and behavior" that constitute the course structure according to the goal. Based on the integrity of educational goals, Bloom has developed a classification system for educational goals, which divides educational goals 
into three categories: cognitive goals, skills (capabilities) goals, and emotional goals. Then he designed the teaching content and teaching process according to the three goals. Therefore, this paper aims to include the three areas of competence, cognitive and emotional. On the other hand, it is necessary to judge whether the content, teaching form and teaching method of the general education course meet the overall teaching objectives. It is also necessary to judge whether the teaching goals of the general education course are consistent with the overall goal of the school, and whether the goals are not contradictory or conflicting, or compatible with each other. Thus we also increase the achievement of teaching goals as an aspect of the teaching goals.

Goal achievement degree: Whether goals are systematic, hierarchical, scientific, specific, developmental and dynamic, and whether the goals are not contradictory, non-conflicting, and compatible with each other.

Goal of competency aspect: to help students master the thinking, methods and basic skills of the subject, and to teach students to use the subject knowledge to analyze other subject or social issues.

Goal of the knowledge aspect: to enable students to acquire knowledge that is conducive to improving the general quality of students, and to let students understand the relationship between this knowledge and other subject knowledge, to provide students with a variety of ways of thinking and understanding of history, understanding of society and the world. Education to form a broad knowledge base and a balanced knowledge structure.

Goal of the emotional aspect: to guide students to form certain interests, attitudes and values, to correctly understand various social phenomena, and to live in harmony with nature, society and others.

\subsection{Evaluation of the Implementation Process of General Education Courses}

In the implementation process, we must consider the teaching goals, teaching methods, teaching effects, etc., which should cover teaching content, teaching arrangements, teaching management, and student feedback. It is necessary to evaluate whether the teaching goals are specific and comprehensive, and whether they are scientific and hierarchical; It is necessary to evaluate whether the teaching goals have taken account the relationship between educational activities and realistic goals, whether they can reflect the individual differences of students; It is necessary to evaluate whether teaching content can reflect the teaching goals of general education, whether it can truly have a full impact on students; It is necessary to evaluate whether teaching method focuses on the transfer of skills and experience, whether it can enlighten thinking, actively mix and match knowledge in various fields; It is necessary to evaluate whether teaching effect reaches teaching goal, achieves the all-round development of students, establishes a perfect world view, values, and outlook on life for students.

\subsection{Evaluation of the Assessment Method of General Education Courses}

A reasonable and effective assessment can reflect the students' comprehensive knowledge and skill level. It is more conducive to the cultivation of application-oriented talents with innovative spirit only by adopting flexible and diverse assessment methods. The general course should pay more attention to the performance evaluation and the formation of students' interest and accomplishment. We should also pay attention to the diversity of assessment forms, including whether to use learning reports, compositions, speeches, operations, experiments, data collection, works display, etc. to assess students.

\subsection{Evaluation of Teaching Effect of General Education Courses}

The evaluation includes four aspects: cognitive domain goal achievement, emotional domain goal achievement, ability domain goal achievement and teaching quality monitoring, so as to comprehensively reflect the achievement of ability domain, cognitive domain, emotional domain goal and the satisfaction of teaching effect.

Table 1. Evaluation Index System of Teaching Quality of Liberal Undergraduate Education.

\begin{tabular}{|c|c|c|c|}
\hline First level index & Second level index & Number & Third level evaluation index \\
\hline \multirow{9}{*}{ Teaching goal } & Goal reaching degree & $x_{1}$ & Whether are goals systematic, hierarchical, scientific, specific, developmental and dynamic? \\
\hline & & $x_{2}$ & Whether are goals compatible with each other, not contradictory, and not conflicting? \\
\hline & Competence field & $x_{3}$ & $\begin{array}{l}\text { Do students master the thinking, methods and basic skills of the subject, and can they use the } \\
\text { knowledge to analyse related issues in the subject or society. }\end{array}$ \\
\hline & & $x_{4}$ & $\begin{array}{l}\text { Ability to analyse and solve other subject areas and social issues using the knowledge and } \\
\text { methods of this subject }\end{array}$ \\
\hline & Cognitive field & $x_{5}$ & $\begin{array}{l}\text { Students accurately comprehend the scientific facts, concepts and categories, principles, laws and } \\
\text { doctrines involved in the course. }\end{array}$ \\
\hline & & $x_{6}$ & $\begin{array}{l}\text { Whether does the goal fully consider the realistic feasibility of educational activities to achieve } \\
\text { the goal? }\end{array}$ \\
\hline & & $x_{7}$ & $\begin{array}{l}\text { Whether does the goal of general education course fully reflect the philosophy of general } \\
\text { education and the pursuit of value? }\end{array}$ \\
\hline & Emotional field & $x_{8}$ & $\begin{array}{l}\text { Whether does the goal combine the school's general course, social needs and student } \\
\text { development? }\end{array}$ \\
\hline & & $x_{9} \cdots \cdots \cdots \ldots$ & Whether does the goal reflect the spirit of the humanities? \\
\hline $\begin{array}{l}\text { Implementation } \\
\text { process }\end{array}$ & Teaching content & $x_{10}$ & $\begin{array}{l}\text { Whether is teaching content closely related to the teaching goal, and are the breadth and depth of } \\
\text { the content suitable for the actual level of the students? }\end{array}$ \\
\hline
\end{tabular}




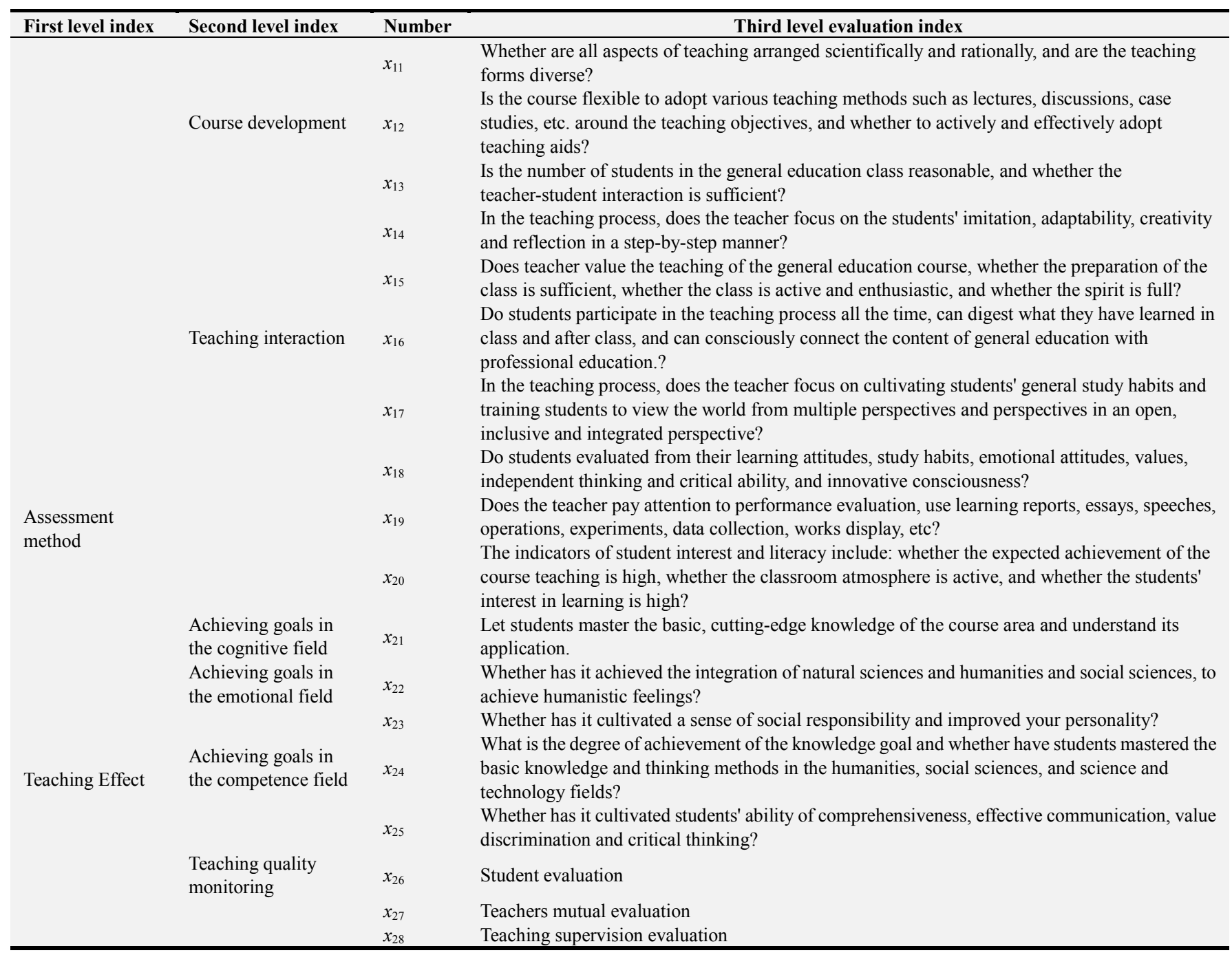

\section{Case Analysis}

Table 2. Experts' scores in various indexes.

\begin{tabular}{llllllll}
\hline \multirow{2}{*}{$\begin{array}{l}\text { Evaluating } \\
\text { indicator }\end{array}$} & \multicolumn{7}{l}{ Young teachers } \\
\cline { 2 - 8 } & $\mathbf{A}_{\mathbf{1}}$ & $\mathbf{A}_{\mathbf{2}}$ & $\mathbf{A}_{\mathbf{3}}$ & $\mathbf{A}_{\mathbf{4}}$ & $\mathbf{A}_{5}$ & $\mathbf{A}_{\mathbf{6}}$ & $\mathbf{A}_{\mathbf{7}}$ \\
\hline $\mathrm{x}_{1}$ & 0.6 & 0.6 & 0.8 & 0.6 & 0.6 & 0.6 & 0.6 \\
$\mathrm{x}_{2}$ & 0.8 & 0.6 & 0.6 & 0.6 & 0.6 & 0.4 & 0.6 \\
$\mathrm{x}_{3}$ & 0.4 & 0.8 & 0.6 & 0.6 & 0.6 & 0.6 & 0.6 \\
$\ldots$ & $\ldots$ & $\ldots$ & $\ldots$ & $\ldots$ & $\ldots$ & $\ldots$ & $\ldots$ \\
$\mathrm{x}_{25}$ & 0.6 & 0.6 & 0.6 & 0.6 & 0.8 & 0.6 & 0.2 \\
$\mathrm{x}_{26}$ & 0.6 & 0.4 & 0.6 & 0.6 & 0.6 & 0.6 & 0.6 \\
$\mathrm{x}_{28}$ & 0.6 & 0.6 & 0.4 & 0.6 & 0.6 & 0.6 & 0.6 \\
\hline
\end{tabular}

In a university, six evaluation experts were organized to evaluate the teaching ability of 7 young teachers in their general courses, which consisted of 3 general education researchers, 2 managers and 1 curriculum manager. According to the opinions provided by the experts, the specific weight data were determined as follows. Each evaluation index is divided into five grades: excellent, good, medium, qualified and unqualified and their corresponding scores are 1, 0.8, 0.6, 0.4 and 0.2 respectively. Seven young teachers were evaluated and scored one by one according to the evaluation criteria by following the class, consulting the teaching plans of young teachers, interviewing students and teachers themselves, and consulting the achievements of teaching and scientific research. The scores of 6 evaluation experts were averaged to yield evaluation index values, as shown in the Table 2 for details.

According to Table 2, the maximum value of each evaluation index is selected as the optimal value to get the reference sequence $x_{0}^{t}(k)$. The evaluation indexes of each teacher were used as the other sequence $x_{i}^{\prime}(k)$. Through the calculation of Eq. (1) (4), a grey correlation coefficient matrix is obtained as

$\left[\begin{array}{ccccccc}0.6 & 1 & 0.42 & & 0.6 & 1 & 1 \\ 0.6 & 0.6 & 0.6 & & 0.6 & 0.6 & 1 \\ 1 & 0.6 & 0.6 & & 0.6 & 1 & 0.6 \\ 0.6 & 0.6 & 0.6 & \cdots \cdots & 0.6 & 1 & 1 \\ 0.6 & 0.6 & 0.6 & & 1 & 1 & 1 \\ 0.6 & 0.42 & 0.6 & & 0.6 & 1 & 1 \\ 0.6 & 0.6 & 0.6 & & 0.33 & 1 & 1\end{array}\right]$

According to Eq. (5), we could obtain grey correlation degree values of teaching ability of $r=(0.7206,0.8370$, 
$0.7417,0.6811,0.7615,0.7312,0.6465)$ for young teachers in general courses. As mentioned above, the evaluation system has five grades: excellent, good, medium, qualified, and unqualified, with corresponding scores are $1,0.8,0.6,0.4$, and 0.2 respectively. Therefore, we can see that the evaluation grades for the seven teachers are all good. Among them, $\mathrm{A}_{2}$ has the best teaching ability while $\mathrm{A}_{7}$ and $\mathrm{A}_{4}$ have relatively poor teaching ability. The ranking result is $A_{2}>A_{5}>A_{3}>A_{6}>$ $\mathrm{A}_{1}>\mathrm{A}_{4}>\mathrm{A}_{7}$.

\section{Conclusion}

In this work, we established a set of evaluation system for the teaching quality of general education courses from four aspects of teaching goals, teaching content, implementation process and assessment methods. According to the Bloom goal classification method, the educational goals are classified into three categories: cognitive, skills (capabilities), and emotional goals. We designed teaching content and teaching process according to the three goals, and increased the achievement of teaching goals, which form an evaluation subsystem of the general goal of teaching together. The indicator system, which is of comprehensive and practical significances, can be used for the evaluation of general education quality in colleges and universities.

The GRA method provides a quantitative assessment for evaluation of general education teaching quality. It can be used to evaluate and comprehensively rank the teaching abilities of young teachers, and find out these aspects to be improved. Teachers can analyze their rankings among peers based on the results of teaching evaluations, and at the same time find out their advantages and disadvantages, which has theoretical and practical significance for improving the quality of general education courses in colleges and universities.

\section{Acknowledgements}

This research was supported by the undergraduate teaching engineering project of Chengdu University of Information Technology (No. BKJX2019069, ZL2018034).

\section{References}

[1] I. Zilic. (2018). "General versus vocational education: Lessons from a quasi-experiment in Croatia, Economics of Education Review," vol. 62, pp. 1-11.

[2] J. T. Ishiyama, K. T. Wingo, S. S. Arteaga, C. M. Hernandez. (2001). "Liberal undergraduate education and reasoning styles: using political scenarios in student assessment," The Social Science Journal, vol. 38, issue 1, Spring, pp. 125-135.

[3] J. Tong, X. P. Zhang. (2012). "Scanning of the Current Situation of General Education Practice in Domestic High-level Universities-Based on the Analysis of Undergraduate Teaching Quality Reports of 39 Universities," New Curriculum Research: Higher Education.
[4] Joshua D. Pitts, John D. Johnson. (2017). "Predicting student success in an undergraduate Sport Management program from performance in general education courses," Journal of Hospitality, Leisure, Sport \& Tourism Education, vol. 21, pp. 55-60.

[5] G. Banister, L. G. Portney, C. Vega-Barachowitz, A. Jampel, M Knab. (2019). "The interprofessional dedicated education unit: Design, implementation and evaluation of an innovative model for fostering interprofessional collaborative practice," Journal of Interprofessional Education \& Practice.

[6] Linda A. Reddy, Gregory A. Fabiano, Christopher M. Dudek, Louis Hsu. (2013). "Instructional and behavior management practices implemented by elementary general education teachers, " Journal of School Psychology, vol. 51, issue 6, pp. 683-700.

[7] Chen Xiangming. (2008). "Exploring the University's General Education Model: Taking the Peking University Yuanpei Project as an example."

[8] Huang Mingdong, Feng Huimin. (2003). "General Education: A New Trend of China's Higher Education Reform," Higher Education Research, vol. 4, pp. 13-16.

[9] Liu Qiyu, Wang Zhi. (2017). "Strengthening the construction of general education curriculum system around the goal of talent training," Contemporary Education Theory and Practice, vol. 9, issue 10, pp. 27-31.

[10] Feng Huimin, Huang Mingdong, Zuo Tian. (2012). "The Teaching Quality Evaluation System and Index Design of General Education in Universities," Educational Research, issue 11, pp. 61-67.

[11] Natalie Förster, Elisabeth Kawohl, Elmar Souvignier. (2018). Short- and long-term effects of assessment-based differentiated reading instruction in general education on reading fluency and reading comprehension, Learning and Instruction, vol. 56, pp. 98-109.

[12] Roki Iwahashi. (2007). A theoretical assessment of regional development effects on the demand for general education, Economics of Education Review, vol. 26, issue 3, pp. 387-394.

[13] Brigita Vainoryte, Birute Zygaitiene. (2015). Peculiarities of Interactive Whiteboard Application during Lessons in Lithuanian General Education Schools, Procedia - Social and Behavioral Sciences, vol. 197, issue 25, pp. 1672-1678.

[14] Fien Depaepe, Johannes König. (2018). General pedagogical knowledge, self-efficacy and instructional practice: Disentangling their relationship in pre-service teacher education, Teaching and Teacher Education, vol. 69, January, pp. 177-190.

[15] Bruce G. Charlton. (2006). Science as a general education: Conceptual science should constitute the compulsory core of multi-disciplinary undergraduate degrees, Medical Hypotheses, vol. 66, issue 3, pp. 451-453.

[16] Alyona Sharunova, Mehwish Butt, Ahmed Jawad Qureshi. (2018). Transdisciplinary Design Education for Engineering Undergraduates: Mapping of Bloom's Taxonomy Cognitive Domain Across Design Stages, Procedia CIRP, vol. 70, pp. 313-318.

[17] Brenda G. Larkin, Karen J. Burton. (2008). Evaluating a Case Study Using Bloom's Taxonomy of Education, AORN Journal, vol. 88, issue 3, September, pp. 390-402. 
[18] Chuanfeng Zheng, Ruiming Li, Mingjun Hu, Linlin Zou. (2019). "Determination of low-temperature crack control parameter of binding asphalt materials based on gray correlation analysis," Construction and Building Materials, vol. 217, issue 30, pp. 226-233.

[19] Tamal Ghosh, Kristian Martinsen, Pranab K. Dan. (2019). "Development and correlation analysis of non-dominated sorting buffalo optimization NSBUF II using Taguchi's design coupled gray relational analysis and ANN," Applied Soft Computing, vol. 85, pp. 105809.
[20] Wang Yunlong, Li Kai, Guan Guan, Yu Yanyun, Liu Fei. (2019)."Evaluation method for Green jack-up drilling platform design scheme based on improved grey correlation analysis," Applied Ocean Research, vol. 85, pp. 119-127.

[21] Huanhuan Li, Diyi Chen, Ehsan Arzaghi, Rouzbeh Abbassi, Silvia Tolo. (2018). "Safety assessment of hydro-generating units using experiments and grey-entropy correlation analysis," Energy, vol. 165, issue 15, pp. 222-234. 